\title{
1. Einleitung - männliche Sorgenkinder
}

Im Gegensatz zu anderen aktuellen Publikationen zum Thema „Mediennutzung von Kindern und Jugendlichen" fokussiert unser Buch vordringlich problematische Mediennutzungsmuster und stellt sich damit in die Tradition zweier in der Medienpädagogik und vielen Bereichen der Medienwissenschaft oft gescholtener Forschungstraditionen: Die Tradition der Medienwirkungsforschung einerseits und die der Kulturpessimisten, Traditionalisten und Bilderstürmer - zumindest sind dies die Titel, die uns bereits verliehen wurden - andererseits. Das mag zum einen mit einer stark kriminologisch geprägten Sicht der Dinge zusammenhängen, die sich in der Regel mit Verhaltensweisen und Einstellungen beschäftigt, die als abweichend und problematisch gelten. Zum anderen vertreten die Autoren dieses Buches die Ansicht, dass Mediennutzung, so individuell unterschiedlich, selbstbestimmt und kompetent sie im Einzelnen auch sein mag, dennoch eigenständige Wirkprozesse entfalten kann.

Der medienwissenschaftliche Diskurs der letzten Jahrzehnte hat dem Nachweis stabiler kausaler Effekte von Mediennutzung hohe methodologische Hürden auferlegt, derer sich die Autoren dieses Buches sehr wohl bewusst sind. Wenn die im Folgenden vorgelegten eigenen empirischen Befunde, die einer Querschnittsbefragung entstammen, dennoch teilweise kausal gedeutet werden, so ist dies immer ein interpretativer Akt und eine bewusste Entscheidung, die andere Wissenschaftler unter Umständen auch anders hätten treffen können. Dadurch, dass wir die hier vorgestellten Ergebnisse in den Kontext der aktuellen internationalen Forschung stellen und kausale Interpretationen unserer Daten mithilfe multivariater Modelle auf ihre Plausibilität prüfen, kann dieses Buch aber vielleicht auch kritischen Lesern neue Denkanstöße geben. Zuerst und vor allem soll dieses Buch aber wissenschaftlich interessierten „Profis“" der Kinder- und Jugendarbeit, Erzieher/innen, Lehrer/innen und Eltern dazu dienen, ihre eigenen Erfahrungen mit den hier vorgestellten Daten in Beziehung zu setzen und unsere Schlussfolgerungen in ihrem täglichen Umgang mit Kindern und Jugendlichen zu berücksichtigen.

Innerhalb dieser Veröffentlichung werden mehrere Dimensionen möglicher Medienwirkungen theoretisch und empirisch aufbereitet. Der erste Punkt ist die Frage der Wirkung von Mediennutzung auf schulische Leistungen von Kindern und Jugendlichen. Bereits in dem PISA zugrunde liegenden Modell zu den Bedingungen schulischer Leistungen ist die Medienumwelt als ein Faktor berücksichtigt, der die individuellen Lernvoraussetzungen eines Schülers beeinflussen kann. Leider wurde in den bisherigen Untersuchungen nach dem PISA-Modell die Medienumwelt nur in unzureichendem Maß operationalisiert, und hier vor allem im Hinblick auf positive Wirkungsvermutungen (Ausstattung der Schulen mit Neuen Medien zur Lernunterstützung) oder im Hinblick auf das kulturelle Kapital im Elternhaus (etwa Anzahl der Bücher im Haushalt). Nach Ansicht der Autoren sollte aber gerade die Alltags- 
mediennutzung von Schülerinnen und Schülern als Kern der medialen Umwelt gesehen werden. Gerade die großen geschlechterspezifischen Unterschiede in der Alltagsmediennutzung bieten möglicherweise neue Erklärungsansätze zu der auch in PISA festgestellten Leistungskrise der Jungen. Diese bildet neben den gefundenen Leistungsunterschieden der Schüler/innen nach sozialer Herkunft und Migration sowie einem Nord-Süd-Gefälle, eine Kernaussage der PISA-Berichte, auch wenn dies in der öffentlichen Diskussion bisher kaum einen Niederschlag fand.

Besonders Jungen, so belegen im Übrigen auch schulstatistische Daten, haben in den vergangenen Jahren im deutschen Bildungssystem deutlich nachgelassen. Dies betrifft vor allem die höheren Bildungsabschlüsse. So zeigen sich zum einen deutliche Geschlechterdivergenzen bei der Verteilung auf die unterschiedlichen Schularten. Jungen besuchen im Vergleich zu Mädchen deutlich häufiger eine Hauptschule und weniger oft das Gymnasium: Im Schuljahr 2005/2006 lag der Jungenanteil an Hauptschulen bei 55,8 Prozent und am Gymnasium bei 46,3 Prozent, an der Realschule war das Geschlechterverhältnis annähernd ausgeglichen mit einem Anteil von 49,8 Prozent männlichen Jugendlichen (Daten Statistisches Bundesamt). Zum anderen dominieren die Jungen auch bei den Wiederholern und Schulabbrechern: Im Schuljahr 2004/2005 war in der Sekundarstufe der Anteil der Jungen an den Wiederholern an Hauptschulen bei 62,5 Prozent, an Gymnasien bei 57,2 Prozent und an Realschulen bei 55,6 Prozent (Insgesamt 58,5 \%). Im selben Schuljahr waren unter den Schulabgängern ohne Hauptschulabschluss 63,6 Prozent männliche Jugendliche zu finden (Daten Statistisches Bundesamt). Bei den Abiturienten haben die Mädchen die Jungen deutlich überholt. Lag der Jungenanteil unter den Abiturienten 1980 noch bei 51,7 und 1990 noch bei 49,6 Prozent, so hat er sich seitdem deutlich verringert und war 1992 bei 47,6 Prozent und im Schuljahr 2004/2005 nur noch bei 43,2 Prozent (Daten Statistisches Bundesamt). Diese Veränderungen können sicherlich nicht ausschließlich auf eine reine Verbesserung der Mädchen zurückgeführt werden. Wir konzentrieren uns daher im vorliegenden Buch auf die Rolle der Alltagsmediennutzung als einen weiteren wichtigen Erklärungsfaktor für dieses Phänomen. Zusätzlich greift das Buch auch die aktuelle Debatte über suchtähnliches Computerspielverhalten auf, da dieser Punkt gerade im Hinblick auf verminderte schulische Leistungen in Zukunft von immer größerer Relevanz sein wird. Auch zu diesem Phänomen werden theoretische Überlegungen und erste empirische Befunde präsentiert.

Nicht nur in Bezug auf die schulischen Leistungen gibt es eine steigende Diskrepanz von Jungen und Mädchen. Sie zeigt sich auch in der in den polizeilichen und kriminologischen Statistiken dokumentierten Entwicklung der Jugenddelinquenz: Der Unterschied in der Kriminalitätsbelastung von Jungen und Mädchen ist seit Mitte der achtziger Jahre beständig angewachsen, wobei die deutlichsten Geschlechterunterschiede in der Gewaltkriminalität zu beobachten sind. Betrachtet man die Entwicklung der Tatverdächtigen unter den 14- bis 18-jährigen Jugendlichen im Bereich der Gewaltkriminalität, so ist eine deutliche Auseinanderentwicklung festzustellen. Im Jahr 1984 lag die Tatverdächtigenbelastungszahl (TVBZ, Tatverdächtige pro 100.000 Einwohner der jeweiligen Altersgruppe) bei den Jungen bei 515 und 
überstieg damit die der Mädchen (TVBZ 57) um 458. Bis zum Jahr 1992 ist die Diskrepanz um fast das Doppelte auf 887 angewachsen (TVBZ Jungen 1.023, Mädchen 136), bis 2006 hat sie sich sogar um mehr als das Dreifache auf 1.511 erhöht (TVBZ Jungen 1.899, Mädchen 388). Dies entspricht für die Jahre von 1984 bis 2006 bei den Mädchen einer Steigerungsquote von 6,8 und bei den Jungen einer Steigerungsquote von 3,7, wobei die Zunahme bei den weiblichen 14- bis 18-jährigen Jugendlichen allerdings auf einem deutlich niedrigeren Niveau zu beobachten ist. Etwa vier Fünftel des in diesem Zeitraum eingetretenen Anstiegs der polizeilich registrierten Jugendgewalt sind damit den Jungen zuzurechnen. Vor diesem Hintergrund wird im zweiten Teil des Buches die Frage nach der Wirkung von Mediengewaltrezeption auf Gewaltprävalenz von Jugendlichen gestellt, wobei auch andere wichtige Erklärungsfaktoren einbezogen werden. Der Konsum gewalthaltiger Medien stellt demnach einen eigenständigen Erklärungsfaktor für die Entstehung von Jugendgewalt dar. 